of CKD stages, there was a statistically significant increase in the thickness of the heart walls and the size of the heart cavities.

We compared echocardiographic parameters of the heart in patients with different stages of CKD and the control group using the Scheffe's test. The patients with stage I CKD differed from the control group by slightly increased EchoCG indices, but the differences were not statistically significant ( $p>0,05$ for all parameters).

Of particular interest were the EchoCG parameters determined among patients with CKD stage III. They showed a significant increase in the thickness of left ventricular posterior wall $(p=0,001)$ and the left ventricle end-diastolic diameter $(p=0,037)$. Changes in the interventricular septum thickness, the size of the left atrium and the left ventricular systolic dimension were not statistically significant $(p>0,05)$.

Patients with CKD stage $\mathrm{V}$ differed in a statistically significant increase in the size of the left atrium, the thickness of left ventricular posterior wall and interventricular septum $(p<0,001)$ and a statistically significant increase left ventricular systolic and diastolic dimensions $(\mathrm{p}<0,05)$.

The analysis showed that the left ventricular mass, left ventricular mass index significantly increased with the progression of CKD stages $(p<0,001)$. The left ventricular mass in patients with $\mathrm{CKD}$ stage $\mathrm{V}$ was significantly increased in 2,3 times in comparison with the control group.

Conclusions Structural-functional changes of the heart in children with CKD manifest left ventricular myocardial hypertrophy in the early stages and the addition of an increase in the left ventricular cavity in patients with CKD stage V.

\section{P49 A CASE OF A STRANGE DYSPNEA}

${ }^{1}$ Antonella Coretti*, ${ }^{1}$ Teresa Merola, ${ }^{1}$ Jessica Cinalski, ${ }^{1}$ Francesca Lotti, ${ }^{1}$ Anna Lanzano ${ }^{1}$ Laura Di Florio, ${ }^{1}$ Valeria Verrotti di Pianella, ${ }^{1}$ Agostino Petraccaro, ${ }^{1}$ Enrica Manca, ${ }^{1}$ Rossella Giorgio, ${ }^{1}$ Rossella Canestrale, ${ }^{1}$ Sofia Siena, ${ }^{1}$ Isabella Patisso, ${ }^{1}$ Loredana Dipasquale, ${ }^{1}$ Michaela Sciacovelli, ${ }^{1}$ Annarita Pedico, ${ }^{2}$ Antonio Marseglia, ${ }^{2}$ Michele Carmine Sacco, ${ }^{1,2}$ Massimo Pettoello Mantovani. 'Departmen of Pediatrics, University of Foggia, Foggia, Italy; ${ }^{2}$ Department of Pediatrics, IRCCS 'Casa Sollievo della Sofferenza', San Giovanni Rotondo (FG), Italy

\subsection{6/archdischild-2019-epa.404}

Infantile hemangioma is the most common benign tumor of infancy, affecting $1-2 \%$ of infants. Hemangiomas of the airway constitute an even smaller percentage, but their management can be challenging due to the potential for life threatening airway compromise. A Subglottic hemangioma (SGH) makes up $1.5 \%$ of all congenital laryngeal anomalies, it is twice more common in females than males and have been linked to low birth weight and prematurity. It is during the early proliferative phase (1-3 months of life) that patients became symptomatic, developing characteristic stridor which may progress to respiratory distress. In this early stage, a $\mathrm{SGH}$ is often mistaken for a more common condition such as croup. The aim of this case is to underline what a recurrent dyspnea or laryngeal stridor in the first 6 months might hide Case report A 4 months-old-girl who was born at term of natural childbirth (birth weight $2.500 \mathrm{~kg}$-SGA), presented with several weeks of unremitting stridor, substernal retractions. She was diagnosed to have bronchiolitis and she had been hospitalized twice in an another hospital and treated with oral steroids and nebulized racemic epinephrine without significant improvement in her symptoms; Than she had been sent to our hospital. She had intercostal and subcostal retractions. Both lungs had equal contribution to respiration, respiratory sounds were coarse and she had both inspiratory and expiratory stridors which were more obvious on bilateral sibilant rales, and inspiratory phase. She also had wheezing. Laboratory tests, echocardiogram and electrocardiogram were normal. Her follow-up showed that she was not responding to treatment and her respiratory distress was increasing, thus she had a laryngofibroscopy that did not reveal any clear structural abnormalities, and performed a CT scan of the neck, that revealed a laryngeal mass, confirmed by an MRI of the neck, which showed a solid tissues of low intensity on T1-weighted spin-echo images and of hyperintensity on T2-weighted spinecho images $(6 \times 8 \mathrm{~mm})$, compatible with SGH. After her workup was complete, she received an initial dose of propranolol at $0.5 \mathrm{mg} / \mathrm{kg}$, which was increased to $2 \mathrm{mg} / \mathrm{kg}$, and no adverse effects were noted. SGH is a rare but potentially lifethreatening disease. A high index of suspicion is vital for the early, accurate diagnosis of this disease. Propranolol treatment has many advantages, it is non-invasive and it has a low complication rate; thus, the use of propranolol as a first-line treatment for $\mathrm{SGH}$ is proposed.

\section{P50 OUTCOMES OF ATRIOVENTRICULAR VALVE REGURGITATION IN PATIENTS WHO HAD ATRIOVENTRICULAR SEPTAL DEFECT REPAIR SURGERY}

${ }^{1}$ Ayş̧e Güler Eroğlu*, ${ }^{2}$ Esra Pehlivan, 'Levent Saltık, ${ }^{3}$ Uğurcan Sayll.. ${ }^{1}$ Istanbul University Cerrahpaşa Medical Faculty, Department of Pediatric Cardiology, Istanbul, Turkey; ${ }^{2}$ Istanbul University Cerrahpaşa Medical Faculty, Department of Pediatrics, Istanbul, Turkey; ${ }^{3}$ Istanbul University Cerrahpaşa Medical Faculty, Department of Public Health, Istanbul, Turkey

\subsection{6/archdischild-2019-epa.405}

Aim Left atrioventricular (AV) valve regurgitation is the most serious residual lesion after surgical correction of atrioventricular septal defect (AVSD). Despite improvements in surgical techniques, left $\mathrm{AV}$ valve regurgitation continues to be the most serious problem after surgery. In this study, it was aimed to investigate the risk factors of the left $\mathrm{AV}$ valve regurgitation by evaluating clinical and echocardiographic findings of patients with AVSD, retrospectively.

Material and methods In this study, 78 patients were enrolled. Preoperative echocardiographic findings, operation data, postoperative echocardiography findings were recorded.

Results Fifteen patients (19\%) were partial, 26 patients $(33 \%)$ were intermediate and 37 patients $(47 \%)$ were complete type. Forty-seven patients $(60.3 \%)$ were female and 25 patients (32\%) had Down's syndrome. The median age at diagnosis was 6 months ranged from one day to 12 years. The median age at operation was 10 months (3-180 months) and the median weight at operation was $6.8 \mathrm{~kg}(4-49 \mathrm{~kg})$. The total follow-up was a median of 103 months (28 days-268 months). In patients with partial AVSD, there was no significant difference in left AV valve regurgitation in the first, postoperative and last echocardiography $(p>0.05)$. In patients with intermediate and complete AVSD, left AV valve regurgitation was higher in the final echocardiography than in the first echocardiography $(\mathrm{p}=0.007$ and $\mathrm{p}=0.01$, respectively). According to the univariate analysis; age, gender, the presence of Down syndrome, operation type, annuloplasty, and pulmonary hypertension were not associated with moderate or severe left AV regurgitation after surgical repair $(p>0.05)$. Left $A V$ valve 